\title{
Fast control of temporal and spatial coherence properties of microscope illumination using DLP projector
}

\author{
José A. Rodrigo* and Tatiana Alieva \\ Universidad Complutense de Madrid, Facultad de Ciencias Físicas, Ciudad Universitaria s/n, Madrid 28040, \\ Spain.
}

\begin{abstract}
We present a novel technique for coherence engineering of the microscope illumination based on a DLP projector providing fast (millisecond range) switchable both temporal and spatial coherence design. Its performance is experimentally demonstrated for speckle-noise free quantitative phase imaging with different spatial coherence states. Strategies for design and control of the light coherence are discussed.
\end{abstract}

Keywords: Image reconstruction techniques, Coherence and statistical optics, Phase retrieval, Microscopy.

\section{INTRODUCTION}

The coherence properties of light are one of the basis of numerous imaging techniques, for example, ranging from optical coherence tomography to quantitative phase microscopy. ${ }^{1,2}$ Both temporal and spatial coherence of the light play a crucial role in the imaging process and have to be properly considered for the reconstruction of information about the imaged specimen. The control of the coherence state provides relevant advantages such as suppression of speckle noise and cross-talk artifacts in the image by reducing the temporal and spatial coherence. This fact together the optical sectioning capabilities of the partial coherence (e.g. gating effect exploited for inspection of the specimen, etc.) have attracted wide interest in bioimaging applications. In particular, speckle-noise and cross-talk reduction allow for accurate quantitative phase imaging of specimens, see for example. ${ }^{3-7}$ Other relevant advantage in controlling the light coherence state is that one may achieve subwavelength spatial resolution when the specimen is illuminated by an evanescent field with its spatial coherence adjusted at subwavelenght scales. ${ }^{8}$ An illumination or probe beam with adjustable coherence properties according to the standing application opens up new and promising perspectives.

In this work, we present a method and device for design and fast control of temporal and spatial coherence of the illumination beam in the context of optical microscopy imaging. The coherence control of the beam is achieved by using a programmable DLP (digital light processing) projector based on DMD (digital micro-mirror device) and LED (light emission diode) technologies. This kind of device can easily be incorporated in optical microscopes and is cost-effective. This technique has been recently proposed in $^{9}$ and allows for wavelength switchable specklenoise free quantitative phase imaging and also stands for multi-spectral analysis of the specimen. In our case, the amplitude and phase of the beam scattered by the specimen are retrieved by measuring several defocused images used as constraint images in the iterative algorithm reported in, ${ }^{7}$ which takes into account the spatial and temporal coherence characteristics of the illumination. From this field information the thickness of the specimen and its refractive index distribution can be reconstructed.

We underline that several works have demonstrated the use of multimedia projectors for different applications in optical microscopy. ${ }^{10-12}$ For instance, in $^{10}$ a multimedia projector is applied to created a re-configurable condenser-lens diaphragm (thus replacing the mechanical iris) and therefore make easier obtaining multiple contrast-enhanced imaging configurations (such as dark-field, Rheinberg optical-staining and oblique illumination microscopy) of transparent samples in a conventional widefield microscope. While in $^{11}$ the projector has been applied to create a light pattern into the sample plane for selective fluorescence microscopy. DMD-based projectors have also been used for fast structured illumination to obtain image superresolution. ${ }^{12}$ In contrast, here we present a novel application of the DLP technology for coherence engineering of light.

\footnotetext{
*E-mail: jarmar@fis.ucm.es
} 


\section{TEMPORAL AND SPATIAL COHERENCE ENGINEERING}

We considered a DLP projector (LG PA70G, 750 ANSI lumens of power, Texas Instruments DMD) with three LED sources providing quasi-monochromatic RGB (red-green-blue) channels. The experimental setup is sketched in Fig. 1(a), where all optical elements are fixed. Note that the projection lens of the projector is replaced by a 4- $f$ setup $(\times 1$ Keplerian telescope) that images the DMD into a static ground glass diffuser (GGD, Thorlabs N-BK7 glass, 1500 grit) that warrants for light spatially incoherent. The projected image at the GGD is then relayed $(\times 1$ Keplerian telescope) into the back focal plane of the condenser lens (Nikon Plan-Achromat, 1.25 $\mathrm{NA}, \times 100$, Oil). In this way, the cross-spectral density $J(\mathbf{r}, \nu)$ of the illumination beam (at the sample $x-y$ plane), which describes the correlation of the field at two points separated by a vector $\mathbf{r}=(x, y)$, is related to the spectral density of the projected image $I_{s}(\mathbf{r}, \nu)$ through the Fourier transform

$$
J(\mathbf{r}, \nu) \propto \int I_{s}\left(\mathbf{r}^{\prime}, \nu\right) \exp \left[\mathrm{i} k \mathbf{r r}^{\prime} / \mathrm{f}\right] \mathrm{d} \mathbf{r}^{\prime},
$$

according to the Van Cittert-Zernike theorem. ${ }^{13}$ Here $k=2 \pi \nu / c=2 \pi n / \lambda_{0}$ is a wavenumber, $\nu$ is temporal frequency, with $\lambda_{0}$ being the wavelength, $n$ is the refractive index of the condenser lens immersion medium (e.g. oil Cargille Type B, $n=1.51$ ), $\mathrm{f}$ is the focal length of the condenser lens. This optical setup generates uniform illumination beam over the sample, where the spatial coherence state follows the Schell model. ${ }^{7,13,14}$

(a) Microscope, illumination and measurement setups:
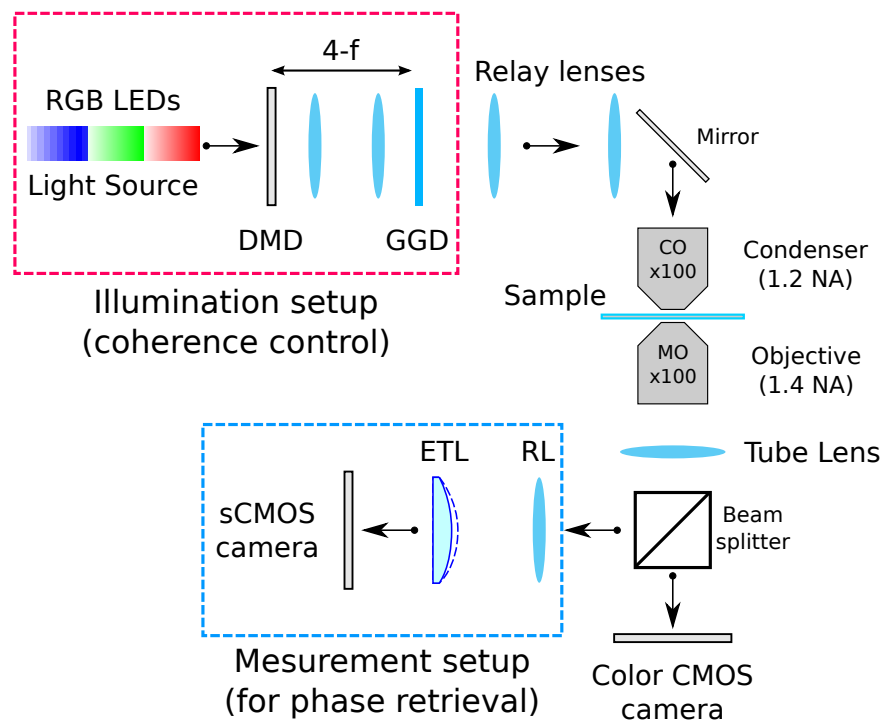

(b)

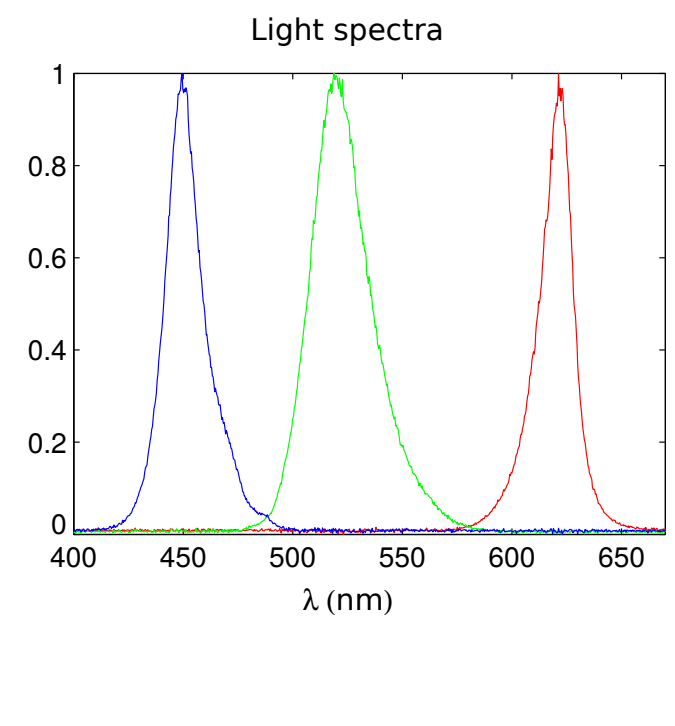

Fig. 1. (a) Sketch of the setup for illumination coherence engineering, transmission microscope and phase retrieval system. Relay lenses (RLs) and tube lens are achromatic doublets with focal length of $150 \mathrm{~mm}$. ETL is a low dispersion varifocal lens electrically controlled by a PC. (b) Measured LED light spectra (Ocean Optics USB2000+ spectrometer).

This system allows for exploring the effects and benefits of coherence sates different from the traditional ones used in imaging. For instance, in the case of an image $I_{s}(\mathbf{r}, \nu)$ corresponding with a circle of radius $a$ (centered at the condenser lens) the complex degree of coherence is given by the well-known Besinc function: $J(\mathbf{r}, \nu)=2 J_{1}(k a r / f) /(k a r / f)$. Other projected images $I_{s}(\mathbf{r}, \nu)$ generate other coherence states $J(\mathbf{r}, \nu)$, where the geometric form of the image $I_{s}(\mathbf{r}, \nu)$, its intensity as well as its color distributions define the illumination coherence and thus the image formation. Note that the temporal coherence is controlled by choosing the appropriate color spectrum of the projected image. Fig. 1(b) displays the measured light spectra of the DLP projector, the central wavelengths are: $\lambda_{0}=622,518$ and $450 \mathrm{~nm}$ with $\triangle \lambda=16,30$ and $20 \mathrm{~nm}$ being the full width at half maximum (FWHM) of the spectrum, respectively. We recall that in the case of quasi-monochromatic LED source $\left(\Delta \lambda \ll \lambda_{0}\right)$ with nearly Gaussian spectrum, the coherence length is often approximated as $l_{c} \approx 0.88 \lambda_{0}^{2} / \Delta \lambda$, see. ${ }^{15}$ Combining three RGB channels a temporally low coherent illumination is obtained, which can be beneficial for 


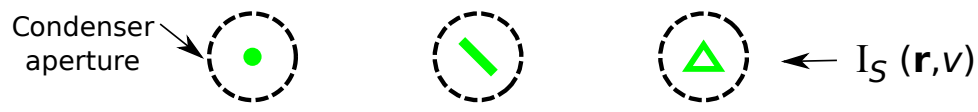

Amplitude of spatial coherence $|J(\mathbf{r}, v)|$
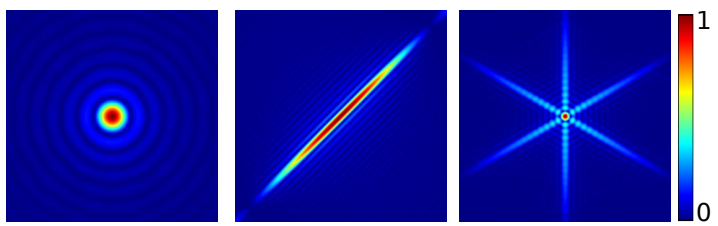

$\lambda_{0}=518 \mathrm{~nm}$

Brightfield images:

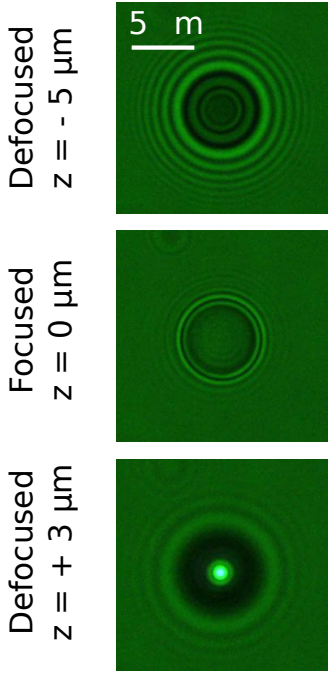

(a)
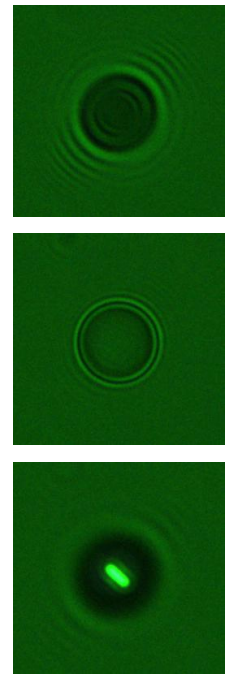

(b)

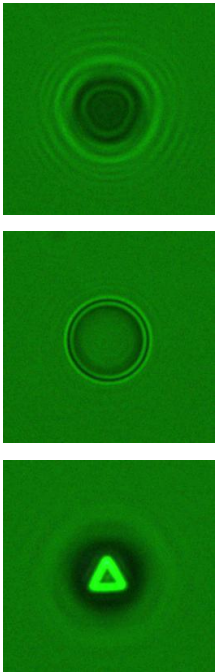

(c)
Thickness profile for:

- $J(r, v)$ in (a)

- $J(r, v)$ in (b)

- $\mathrm{J}(\mathbf{r}, v)$ in (c)

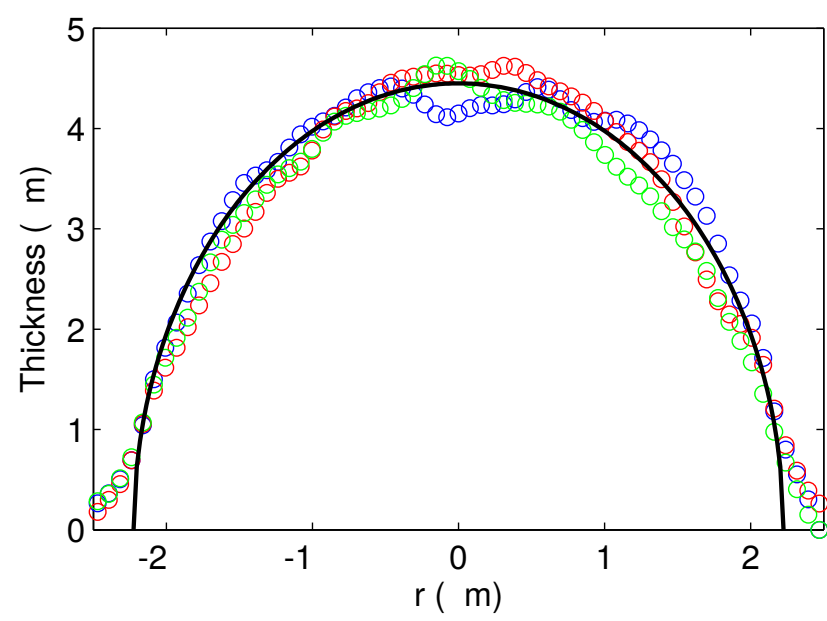

(d)

Fig. 2. (a)-(c) Amplitude of the cross-spectral density $J(\mathbf{r}, \nu)$ associated with three images $I_{s}(\mathbf{r}, \nu)$ projected into the back focal plane of the condenser lens for central wavelength $\lambda_{0}=518 \mathrm{~nm}$. Diffraction patterns measured in brightfield for a polystyrene bead at three focusing distances (in each coherence state) are also shown in (a)-(c). The profile of the reconstructed thickness distributions of the polystyrene bead in the latter coherence states are displayed in (d).

tomographic reconstruction of the sample. ${ }^{6}$ Moreover, by choosing consecutively one of the RGB channels, dispersion analysis of the sample can be performed. This design results in more versatile and faster control of light coherence (in microscopy) than the ones based on: Varying color filters and mechanical diaphragm (iris) of the condenser lens, or alternatively by using partially coherent beams generated from a coherent laser modulated by a moving diffuser or a re-configurable laser cavity. ${ }^{16}$

A brightfield microscope comprising a high numerical aperture objective lens (Olympus UPLSAPO, 1.4 NA, $100 \times$, Oil) and a color camera (Thorlabs-DCC1240C) have been used for image monitoring tasks and qualitative analysis of the sample, see Fig. 1(a). To obtain quantitative phase imaging, several defocused images are measured by using the setup developed in ${ }^{7}$ comprising a fast electrically tunable lens (ETL, Optotune EL-1030-C) and monochrome high-resolution sCMOS camera (Hamamatsu, Orca Flash 4.0, 16-bit gray-level, pixel size of $6.5 \mu \mathrm{m})$, see Fig. 1(a). As an example we considered a sample of polystyrene spheres $(4.7 \mu \mathrm{m}$ bead diameter, Spherotech Lot. AD01) immersed in distiller water. In Fig. 2 the diffraction pattern of a polystyrene sphere (defocused before and after the focal plane $z=0 \mu \mathrm{m}$ ) are shown for three different illumination states corresponding to $I_{s}(\mathbf{r}, \nu)$ : Green circle, rectangle and triangle. The amplitude of spatial coherence is displayed in Fig. 2(a)-(c) for each case. The diffraction patterns reveal the change in the light coherence state caused by the shape of the projected images $I_{s}(\mathbf{r}, \nu)$. Indeed, the pattern is symmetric when $I_{s}(\mathbf{r}, \nu)$ is a centered circle [Fig. 2(a)] and asymmetric in the other cases. As expected, diffraction fringes are well-observed only in the direction corresponding to the smallest side of the projected rectangle image in Fig. 2(b), where the coherence is 


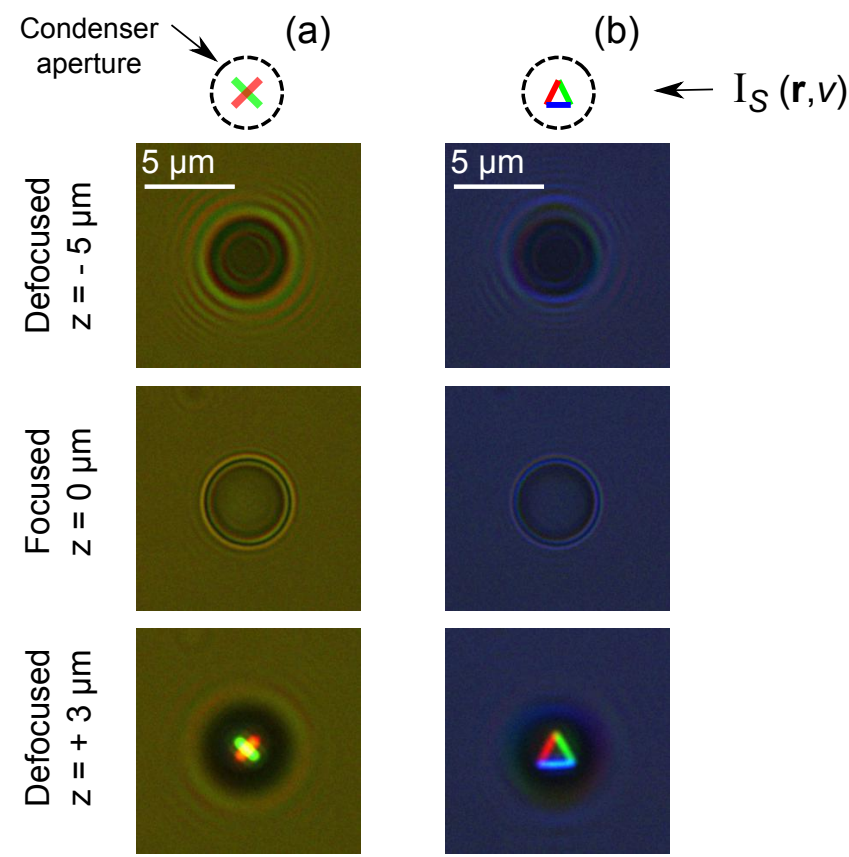

Fig. 3. Diffraction patterns measured in brightfield for a polystyrene bead at three focusing distances and for tow different spatial-temporal coherence states associated to the image images $I_{s}(\mathbf{r}, \nu)$ displayed in top at (a) and (b), respectively. This example illustrates the capabilities of the proposed setup for beam coherence engineering at different wavelengths.

higher. Note that this effect is not observed in the focal plane $(z=0)$ because the intensity distribution at this plane does not depend of the spatial coherence state for the Schell-model illumination. ${ }^{13}$ We also underline that the spherical particle works as a micro-lens that re-imaged $I_{s}(\mathbf{r}, \nu)$ at its focal plane as observed in the fourth row of Fig. 2(a)-(c). To illustrate the versatility of the proposed approach, two different spatial coherence states comprising multiple wavelengths are shown in Fig. 3. This combination of colors and spatial coherence states could be useful to study the wavelength-dispersion properties of a sample and its optical inspection apart from quantitative imaging studies (e.g. thickness distribution reconstruction).

\section{QUANTITATIVE PHASE IMAGING WITH THE CONTROLLED ILLUMINATION COHERENCE STATES}

In the case of partially coherent light the reconstruction of the phase delay caused by the specimen is more complex than in the case of coherent light microscopy. ${ }^{2}$ Such a phase retrieval problem has been studied in detail in the context of electron microscopy and X-ray imaging by means of non-interferometric methods such as transport of intensity equation (TIE) and iterative algorithms. ${ }^{17-22}$ The developed techniques can be extended to the visible wavelength range to obtain quantitative phase imaging with a brightfield microscope under Khöler illumination, see. ${ }^{5,7,9,23-25}$ For instance, inspired by the method proposed in, ${ }^{21}$ we have developed an iterative technique that is able to retrieve both the phase delay caused by the object and degree of spatial coherence of the microscope illumination beam. ${ }^{7}$ Note that, due to the loss of both temporal and spatial coherence, the phase retrieval using interferometric methods is much difficult in practice.

Our approach requires the measurement of at least four defocused images at the detector plane [e.g. sCMOS camera in the measurement setup displayed in Fig. 1(a)]. Specifically, each defocused image is given as a function of the ETL focal length, $\mathrm{f}_{m}$, and coherence sate as it follows:

$$
I_{P C}^{m}(\mathbf{r}, \nu) \propto \int I_{C}^{m}\left(\mathbf{r}^{\prime}, \nu\right) I_{s}\left[\left(\mathbf{r}-\mathbf{r}^{\prime}\right) S_{m}, \nu\right] \mathrm{d} \mathbf{r}^{\prime}
$$


see $^{7}$ for further details. Here, $S_{m}=\mathrm{f}_{m} /\left(d-\mathrm{f}_{m}\right)$ is a scaling factor where $d$ is a fixed distance between the ETL and the sCMOS camera. The expression Eq. (2) is the convolution between $I_{s}(\mathbf{r}, \nu)$ and $I_{C}^{m}(\mathbf{r}, \nu)$ that corresponds to the intensity distribution which would be obtained using ideal (speckle-free) spatially coherent illumination. The measurement is performed inside the coherence gate according to the value of coherence length, in our case $l_{c} \sim 9 \mu \mathrm{m}$ for $\lambda_{0}=518 \mathrm{~nm}$. Note that as the coherence becomes low the defocusing range in the measurement has to be shorter. In this range the object's diffraction patterns have to be well resolved and sufficiently vary with defocus such that the iterative algorithm can recover the sample phase information. ${ }^{7,19}$ Fig. 2(b) displays the profile of the bead's thickness distribution obtained from the retrieved phase information for each configuration of spatial coherence considered in Fig. 2(a)-(c). Here we have used the same procedure as reported in ${ }^{7}$ (see Section 2) and images $I_{P C}^{m}(\mathbf{r}, \nu)$ of $1024 \times 1024$ pixels, the convergence is typically reached after 10 iterations in a few seconds $(20 \mathrm{~s})$. These experimental results are in good agreement with the expected thickness profile of a spherical bead and demonstrate that quantitative phase imaging is possible even using partially coherent illumination whose spatial coherence exhibits rather different symmetry with respect the conventional circular one. ${ }^{9}$

\section{CONCLUSIONS}

The studied examples illustrate the versatility of the developed illumination setup for light coherence control in optical microscopy and in particular for quantitative phase imaging. This setup comprises three color LED sources (RGB) and a micromirror-based spatial light modulator (DMD) providing a programmable and fast performance in the millisecond range. Control of temporal (e.g. by changing the LED color) and spatial coherence (e.g. by varying the pattern displayed into the DMD) according to the standing application brings promising advantages due to speckle-noise free and optical gating effect (axial image sectioning). Fast control of light coherence opens up new perspectives for in vivo inspection and quantitative analysis of samples, for example multilayered cell structures. It can be useful for analys of information at subwavelent spatial resolution when using an evanescent illumination field. ${ }^{8}$

\section{ACKNOWLEDGMENTS}

Spanish Ministerio de Economía y Competitividad is acknowledged for funding the project TEC2011-23629.

\section{REFERENCES}

1. Boas, D., Pitris, C., and Ramanujam, N., [Handbook of Biomedical Optics], Taylor \& Francis (2011).

2. Ferraro, P., Wax, A., and Zalevsky, Z., [Coherent Light Microscopy: Imaging and Quantitative Phase Analysis], Springer Series in Surface Sciences, Springer (2011).

3. Paganin, D. and Nugent, K. A., "Noninterferometric phase imaging with partially coherent light," Phys. Rev. Lett. 80, 2586-2589 (1998).

4. Langehanenberg, P., Bally, G. v., and Kemper, B., "Application of partially coherent light in live cell imaging with digital holographic microscopy," J. Mod. Optic 57(9), 709-717 (2010).

5. Phillips, K. G., Velasco, C. R., Li, J., Kolatkar, A., Luttgen, M., Bethel, K., Duggan, B., Kuhn, P., and McCarty, O., "Optical quantification of cellular mass, volume and density of circulating tumor cells identified in an ovarian cancer patient," Frontiers in Oncology 2(72) (2012).

6. Kim, T., Zhou, R., Mir, M., Babacan, S. D., Carney, P. S., Goddard, L. L., and Popescu, G., "White-light diffraction tomography of unlabelled live cells," Nat Photon (3), 256-263 (2014).

7. Rodrigo, J. A. and Alieva, T., "Rapid quantitative phase imaging for partially coherent light microscopy," Opt. Express 22(11), 13472-13483 (2014).

8. Baleine, E. and Dogariu, A., "Variable coherence scattering microscopy," Phys. Rev. Lett. 95, 193904 (2005).

9. Rodrigo, J. A. and Alieva, T., "Illumination coherence engineering and quantitative phase imaging," Opt. Lett. 39(19), 5634-5637 (2014).

10. Samson, E. C. and Blanca, C. M., "Dynamic contrast enhancement in widefield microscopy using projectorgenerated illumination patterns," New Journal of Physics 9(10), 363 (2007).

11. Stirman, J. N., Crane, M. M., Husson, S. J., Gottschalk, A., and Lu, H., "A multispectral optical illumination system with precise spatiotemporal control for the manipulation of optogenetic reagents," Nat. Protocols 7(2), 207-220 (2012). 
12. D. Dan et al., "DMD-based LED-illumination Super-resolution and optical sectioning microscopy," Sci. Rep. 3 (2013).

13. Born, M. and Wolf, E., [Principles of Optics], Cambridge University Press, Cambridge (1999).

14. Schell, A. C., The multiple plate antenna, PhD thesis, Massachusetts Institute of Technology (1961).

15. Goodman, J. W., [Statistical Optics], Wiley\&Sons, NY (2000).

16. Nixon, M., Redding, B., Friesem, A. A., Cao, H., and Davidson, N., "Efficient method for controlling the spatial coherence of a laser," Opt. Lett. 38(19), 3858-3861 (2013).

17. Teague, M. R., "Deterministic phase retrieval: a Green's function solution," J. Opt. Soc. Am. 73(11), 1434-1441 (1983).

18. Gureyev, T. E. and Nugent, K. A., "Rapid quantitative phase imaging using the transport of intensity equation," Opt. Commun. 133(1-6), 339-346 (1997).

19. Martin, A. V., Che, F. R., Hsieh, W. K., Kai, J. J., Findlay, S. D., and Allen, L. J., "Spatial incoherence in phase retrieval based on focus variation," Ultramicroscopy 106, 914-924 (2006).

20. Burvall, A., Lundström, U., Takman, P. A. C., Larsson, D. H., and Hertz, H. M., "Phase retrieval in X-ray phase-contrast imaging suitable for tomography," Opt. Express 19(11), 10359-10376 (2011).

21. Clark, J., Huang, X., Harder, R., and Robinson, I., "High-resolution three-dimensional partially coherent diffraction imaging," Nat Commun 3, 993 (2012).

22. Bravin, A., Coan, P., and Suortti, P., "X-ray phase-contrast imaging: from pre-clinical applications towards clinics," Physics in Medicine and Biology 58(1), R1 (2013).

23. Barty, A., Nugent, K. A., Paganin, D., and Roberts, A., "Quantitative optical phase microscopy," Opt. Lett. 23(11), 817-819 (1998).

24. Jingshan, Z., Tian, L., Claus, R. A., Dauwels, J., and Waller, L., "Partially coherent phase recovery by Kalman filtering," in [Frontiers in Optics 2013 Postdeadline], Frontiers in Optics 2013 Postdeadline , FW6A.9, Optical Society of America (2013).

25. Jingshan, Z., Tian, L., Dauwels, J., and Waller, L., "Partially coherent phase microscopy with arbitrary illumination source shape," in [Classical Optics 2014], Classical Optics 2014, CTu1C.5, Optical Society of America (2014). 